\title{
Hong Kong Student Science Project Competition 2021
}

10.17975/sfi-2021-007

The Hong Kong Student Science Project Competition (HKSSPC) promotes the interest in science and technology among youth, develops their creativity and critical thinking skills through an innov

ative application of science and technology, and ignites their passions and career interests in these areas.

This year, the HKSSPC was organized by The Hong Kong Federation of Youth Groups, the Education Bureau, the Hong Kong Science Museum, and the Hong Kong Science and Technology Parks Corporation. Furthermore, it was supported by the Innovation and Technology Commission and the Hong Kong Young Academic of Sciences. We extend our thanks to all these groups for making this year's competition a success.

STEM Fellowship collaborated with the HKSSPC Secretariat to provide youth from Hong Kong with the unique opportunity to submit their work in the STEM Fellowship Journal. This year's theme was "Inspiration from Living - Innovation from Science" with an emphasis on United Nations' Sustainable Development Goals. The broad scope of the competition allowed participants to submit their work in a variety of areas such as water pollution, nanoparticles, artificial intelligence systems, agriculture, environmental health, plastics, waste reduction, and many more.

We are pleased to share the creativity and ambitious drive for research demonstrated by HKSSPC's participants in these proceedings. We would like to congratulate every passionate individual who participated in the HKSSPC this year and wish them the best in their future STEM-related endeavours.

Abhinand Thaivalappil \& Ruijia Zhang

Co-Managing Editors, STEM Fellowship Journal

\section{Gurman Khera}

Science Communication Chief Officer, STEM Fellowship

\section{Dr. Sacha Noukhovitch}

Founder, President and Editor-in-Chief

STEM Fellowship/STEM Fellowship Journal

\section{Disclaimer}

The abstracts contained in this document were submitted to HKSSPC 2021 by student teams. The STEM Fellowship Journal editorial board has made every effort to ensure proof and English editing of these abstracts in a limited amount of time, and neither organization as a whole or any of its volunteer members can be held accountable for inaccuracies that may have occurred in the abstract publication. Abstracts are published in no particular order. 


\section{Anti-bacterial crab bio-bandages with bio- dressings $\mathbf{2 . 0}$}

\section{OR Hiu Ying, LI Hiu Fung, YIU Long, SO Yan Wing, LI Lok Yi Happy \\ Carmel Pak U Secondary School}

Commercially available bandages such as hydrocolloids are neither biodegradable nor anti-bacterial. However, crab shells are made up of about $25 \%$ to $30 \%$ of chitin and they could be transformed into chitosan and hydrogel which are known to be anti-bacterial (Hasan, 2018; Chao, 2019), haemostatic (Okamoto, 2003; Hu, 2018), bio-degradable, and have good water absorbance. Roasted crab hydrogels are even waterproof, so they are potential alternatives for anti-bacterial bio-bandages with bio-dressings. We obtained crab hydrogel bio-dressings and roasted crab bio-bandages by demineralizing crab shells to chitin using dilute nitric acid, deacetylation of chitin to chitosan using concentrated $\mathrm{NaOH}$, forming hydrogel using vinegar and roasting hydrogels at $120^{\circ} \mathrm{C}$ for 30 mins in the oven. The degree of deacetylation of DD\% of crab hydrogels and roasted crab hydrogels determined by FTIR spectrum II were $82.6 \%$ and $72.2 \%$ respectively, so they could serve as haemostatic agents (due to chitosan). Biodegradation was done in soil. Roasted crab hydrogel and crab hydrogel took 42 days and a month for complete biodegradation, respectively. Crab hydrogels and roasted crab hydrogels showed a significant anti-bacterial effect in solutions with oral bacteria, as no bacterial colonies were found in these samples with different dilutions. They met the requirement of serial no.204 in IS997:2004. Furthermore, in the FSA Free-Swell Absorbency test, crab hydrogel biodressing absorbed 1.86g synthetic blood per dressing which was much higher than that of commercial hydrocolloid (0.299g) based on BS EN 13726-1. Crab bio-bandages with crab bio-dressings should be eligible for marketing.

Supervisors: IP Yuen Yu, WONG Tsz Yeung

\section{Synthesis of nanoparticles by chemical method for antibacterial bioplastics}

\section{ZHANG Lok Hai, TANG Cheuk Man \\ St. Paul's Convent School}

During the pandemic, plastic covers are used on lift buttons for preventing the spread of germs. However, these covers are not biodegradable, and require frequent disinfection. Therefore, the goal of this project was to develop antibacterial nanoparticles(NPs)-containing bioplastic as a better alternative. Silver, copper, and nickel nanoparticles were successfully synthesized by simple chemical method from their salt using sodium borohydride or vitamin C as a reducing agent. Sodium alginate and starch were found to be effective stabilising agents for AgNPs and CuNPs, respectively. The size of the synthesized nanoparticles was measured by dynamic light scattering (DLS). The average diameter of AgNPs was found to be about $18-30 \mathrm{~nm}$ and the solution could stay yellow for months without any formation of precipitate. The nanoparticles synthesized were then mixed with the sodium alginate or corn-starch solution to produce bioplastics sheets. The antibacterial abilities and mechanical strength of the bioplastics were tested by agar plates and spring balance, respectively. The areas of the agar plates, which were covered with NPs-containing bioplastics, showed no bacterial colonies, proving that they can inhibit the growth of bacteria. Also, more than half of the bioplastic samples were found to have a breaking force greater than that of the plastic wrap. Nanoparticles aggregate easily to give large particles without a good stabilizing agent. This project has developed a convenient approach to synthesize nanoparticles that could be used directly to produce bioplastics sheets without further separation. This approach could minimize the loss and help to reduce the size of the nanoparticles to give an effective antibacterial effect.

Supervisor: Charlotte PO

\section{Bi-I-See}

\section{NGIE Ching, LAW Tsz Yan, HUNG Yui}

\section{Kit Sam Lam Bing Yim Secondary School}

In recent years, many people have taken up cycling as a hobby. However, the number of bicycle accidents has also increased. Bi-I-see is a smart bicycle device that uses ultrasonic distance sensing technology to help cyclists accurately assess the required stopping time. Moreover, using object recognition technology, cyclists can pay more attention to the condition of the traffic from the rear to avoid entering the blind spot area of large vehicles, thereby reducing bicycle accidents. Function 1: Obstacle ahead warning. The ultrasonic 
distance sensor detects the distance between the bicycle and the obstacle ahead. When the distance is shorter than the distance required for the cyclist to apply the brakes properly, the Micro:bit will turn on the buzzer immediately. It reminds the cyclists to stop the bicycle. The speed of the bicycle is measured by the Hall sensor installed on the wheel. The safety distance can then be determined based on the speed of the bicycle. Function 2: Rear traffic warning. When the artificial intelligence vision sensor identifies a large vehicle at the back of the cyclist, Micro:bit will switch on the buzzer, which reminds the cyclist to let the large vehicle pass or stay in front of it, thereby avoiding accidents because of turning left at the same time. To avoid bicycle accidents on cycling tracks or roads, the driving attitude of the cyclist is the most important. However, we hope that the smart bicycle device $\mathrm{Bi}$ I-See can help cyclists stay alert and reduce potential danger. Supervisor: CHAN Wai Ping

\section{Test for heavy metals with brine shrimp}

\section{LUK Choi Yin Jessica, FU Ka Wing, MO Hoi Ki, KAN Ka Wai, LEE Sze Yuen}

\section{Queen Elizabeth School Old Students' Association Secondary} School

The use of brine shrimp nauplii to test for the overall toxicity of environmental samples is proposed. Brine shrimp nauplii were cultured with different concentrations of heavy metals, including chromium (III), copper (II), nickel, lead, zinc, and organic pollutants, including triclosan, oxybenzone, octinoxate, and bisphenol A. The activity of the brine shrimp nauplii was observed under a dissection microscope to determine the death rate. Results showed that brine shrimp nauplii are very sensitive to cadmium, bisphenol $A$, and oxybenzone. The LC50s (24h) are 24.9, 13.4 and 8.4 ppm, respectively. Zinc is likely to have a synergistic toxic effect with nickel or lead. The synergistic toxic effects of other heavy metals and organic pollutants should be confirmed with further investigation. Brine shrimp nauplii were treated with extracts from three different sediment samples collected from the oyster culture zone of the Deep Bay. The sediment samples were extracted with neutral sodium acetate to dissolve the exchangeable heavy metal ions and some organic pollutants. The death rate of brine shrimp nauplii treated with the sediment extract of Hang Hau Tsuen was similar to
$1 \mathrm{ppm}$ PBA. It was also about 10 to $20 \%$ higher than that of the other two sites (Pak Nei and Sha Kiu Tsuen). Since Hang Hau Tsuen is closer to the residential area and Lau Fau Shan Seafood Market than the other two sites, its sediment sample is likely to have a higher level of environmental pollutants. The results suggest that brine shrimp nauplii may be used as a biomarker to monitor the environmental changes in the overall level of pollutants in sediment samples.

Supervisor: CHAN Pat Chun

\section{Driver drowsiness detection system}

\section{LOUIE Ho Shun, TIN Hin Wing, TSANG Chung Ho, WONG Chun Ting, WONG Lo Yi \\ Heung To Secondary School (Tseung Kwan O)}

Drivers in Hong Kong are sometimes drowsy due to long working hours. Although devices are available to wake up drivers, their effectiveness is insufficient. We developed a device called "Driver Drowsiness Detection System" which uses computer vision technology to monitor drivers' status and ensure the safety of people. The system is made of a Raspberry Pi 3 microcontroller, web camera, LEDs, LCDdisplay and loudspeakers. When the driver is drowsy, LEDs will turn $\mathrm{ON}$ and the LCD-display will show a warning signal for passengers (including visually impaired and hearing-impaired passengers). The voice system will also inform the passengers that the driver is drowsy and ask passengers to wake up the driver to avoid an accident. Emails will be sent immediately to the transport company to record the driver's drowsiness data. The transport company can analyze the data and reschedule the rest time of the driver to ensure that no driver works in a tired state. Our repeated tests showed that the system detects the driver's drowsiness with 95\% accuracy. Even if the driver wears a mask or glasses, the system can still monitor the driver's state. Collectively, passengers can travel safely if this system is installed on public transportation. Drivers' safety awareness and service performance can be enhanced through this system. Moreover, the system is automated and will not increase the workload of drivers.

Supervisor: WONG Tung Shek

\section{Nanocellulose from sugar cane bagasse for removing aqueous heavy metal ions}




\section{POON Wing Yu, HUI Shing Chun, WAN Yau Long, HONG Hang, LEE Ying Yi}

Wong Shiu Chi Secondary School

Dry sugarcane bagasse was delignified by reacting with $5 \%(w / w) \mathrm{NaOH}_{(\mathrm{aq})}$ while stirring at $70^{\circ} \mathrm{C}$ for $1 \mathrm{~h}$, and then bleached by treatment with $4 \%(w / w) \mathrm{NaOH}_{(\mathrm{aq})}$ and $24 \% \mathrm{H}_{2} \mathrm{O}_{2 \text { (aq) }}$ while stirring at $50^{\circ} \mathrm{C}$ for 2 hours to extract the pretreated cellulose. The resulting pretreated cellulose was changed into nitro-oxidized nanocellulose (NO-NCS) by stirring with $65 \% \mathrm{HNO}_{3(\mathrm{aq})}$ and sodium nitrite at $50^{\circ} \mathrm{C}$ for 3 hours and then at room temperature for 2 days. The overall percentage yield of the conversion of sugarcane bagasse into (NO-NCS) was $16.77 \%$. The absorption capacity of the prepared NO-NCS for five types of heavy metal ions: $\mathrm{Cu}^{2+}{ }_{(\mathrm{aq})}{ }^{\prime}$ $\left.\mathrm{Cr}^{3+}{ }_{(\mathrm{aq})}, \mathrm{Mn}^{2+}{ }_{(\mathrm{aq})}\right)^{\mathrm{Pb}^{2+}{ }_{(\mathrm{aq})}}$ and $\mathrm{Hg}^{2+}{ }_{(\mathrm{aq})}$, ranging from 0-10 mins to 0-35 mins and the average rate of absorption for them by NO-NCS ranging from 0-63 s to 0-660 s were found to range from 0.0030 to $0.096 \mathrm{mmol} / \mathrm{g}$ and from $4 \times 10^{-9}$ to $2.9 \times 10^{-7} \mathrm{~mol} /$ $\mathrm{g} \cdot \mathrm{s}$, respectively, as well as both have the same decreasing order of $\mathrm{Cu}^{2+}{ }_{(\mathrm{aq})}>\mathrm{Cr}^{3+}{ }_{(\mathrm{aq})}>\mathrm{Mn}^{2+}{ }_{(\mathrm{aq})}>\mathrm{Pb}^{2+}{ }_{(\mathrm{aq})}>\mathrm{Hg}^{2+}{ }_{(\mathrm{aq})}$. Experiments also showed that the average rate of absorption generally decreased with time. The very low absorption capacity of NO-NCS for the metal ions in a short period of time may be attributed to the low rate of absorption as a result of very slow diffusion of surface adsorbed metal ions to the interior of the nanocellulose crystals.

Supervisor: CHU Hon Yue

\section{Forma-Rid chitosan gel}

\section{CHOI Yau Nam, NG Kin Kwan, TSUI Ka Yu, FUNG Yeuk Sin, WONG Yee Mei \\ Carmel Pak U Secondary School}

Formaldehyde is found in resins used in the manufacture of composite wood products such as wardrobes, glues, permanent press fabrics, paints and coatings, lacquers and finishes, and paper products (1). Formaldehyde may cause eyes, trachea, and skin irritation. High levels of exposure to formaldehyde may cause myeloid leukemia and cancers (2). In this study, chitosan gel is prepared by dissolving in vinegar and used to remove formaldehyde by breaking it down into water. In the experiment, the effect of $0.1 \mathrm{~g}-0.5 \mathrm{~g}$ of chitosan gel on formaldehyde removal was tested. The concentration of chitosan for achieving the optimal efficiency was $0.2 \mathrm{~g}$ per $10 \mathrm{~mL}$ of vinegar. The efficiency of $0.2 \mathrm{~g}$ of chitosan gel was tested by putting it into different concentrations of formaldehyde, including 1:20, 1:40, 1:60, 1:80 and 1:100. The result showed that $0.2 \mathrm{~g}$ of chitosan gel can remove $99.7 \%$ of formaldehyde with the dilution of 1:20. The efficiency of our chitosan gel was also compared with commercially available products including ion air purifier, formaldehyde remover, charcoal powder, and charcoal beads. Chitosan gel was found to be the most effective among all products and removed the highest amount of formaldehyde (99.04\%) with the dilution of $1: 100(3,4)$. The gel was found to be reusable 2-3 times with similar efficiency. Moreover, it only costs 20 cents, which is a very low cost, and is feasible in mass production. In summary, our chitosan gel was found to be a cost-effective and ecofriendly product with high removal efficiency.

Supervisor: WONG Tsz Yeung

\section{ACE fertilizer}

TSO Zi Xin Cecilia, LEUNG Nga Wun Sharlene, CHEUNG Ka Hin Kyan, ZAK Leila, IP Jeovana Claire Victoria Shanghai Academy

Upon seeing the vast amount of coffee residue in bins and learning about the 23 million tons of coffee residue produced as waste per year, we were alarmed by the increasing problem of food waste. We devised a solution to transform common household food waste, particularly agar, coffee residue, and eggshells, into ACE fertilizer - an effective, natural, and practical alternative to commercial fertilizers. Agar serves as an effective binding agent and slow-release fertilizer with the ability to form a thermo-reversible gel-like substance in $1 \%$ agar solution. Coffee residue's mineral ions and phosphodiesterase enzymes promote healthy growth and pest deterrence in plants. Eggshells counter the acidity of coffee residue and provide an optimal calcium content for plants. ACE was synthesized by boiling the ingredients in the ratio 1(agar):100(water):1(coffee residue):3(eggshells), then tested with the parameters of growth rate, leaf greenness, biodegradability, soil $\mathrm{pH}$, and dry mass. The results show: (i) ACE increases plant growth by $120 \%$, promoting a similar growth rate to commercial fertilizers, (ii) ACE promotes an optimal chlorophyll content for plants, increasing photosynthesis rate, providing additional energy for growth, (iii) ACE can biodegrade within 10 days, being eco-friendly, (iv) 
the $\mathrm{pH}$ of soil with ACE lies within the optimal range for plant growth (pH 5.5 to 7), and (v) plants in ACE have the highest dry mass of $1.2 \mathrm{~g}$, accumulating the most organic material. Ultimately, ACE's slow-release feature promotes stable growth while being biodegradable, and thus is an effective and ecological solution for mitigating the food waste problem. Supervisor: Yuki WONG

\section{Kombuchas of putrescibles as bio-disposables 2.0}

\section{LEUNG Wai Chung, YIP Tsz Ho, NG Ka Ho, CHI Yui lam, LEUNG Chun Ni \\ Carmel Pak U Secondary School}

In the transition from disposable plastics to biodegradable alternatives, roasted kombucha straws and cups made of fruit skins are plausible options as they are biodegradable and durable. Kombuchas are fermented products of condensed tannins which are known for their polymerization chemistry. Tannin content was obtained using vanillin- $\mathrm{HCl}$ tests and relative amount of hydroxyl groups and physical properties of roasted kombucha were obtained based on FTIR Spectrum II. Tests were also done based on international standards to justify the marketing of kombucha disposables. Results were as follows: (i) when the time of roasting of kombuchas of lemon skins was between 0 to $15 \mathrm{mins}$ at $120^{\circ} \mathrm{C}$, the ratio of absorbance of $-\mathrm{OH}$ to the absorbance of $\mathrm{C}=\mathrm{C}$ in FTIR dropped significantly from 1.65 to 1.24 , the percentage by mass of water absorbed significantly dropped by $33 \%$ and the strength of the kombucha obtained was the strongest among other kombuchas. These results indicated that structural changes of kombuchas of lemon skins took place when kombuchas of lemon skins were roasted at $120^{\circ} \mathrm{C}$ for 0 to 15 minutes; (ii) for complete biodegradation, roasted kombucha (lemon skins) took 2 to 3.5 weeks; (iii) no bacterial colonies were present in drinking water kept overnight in roasted kombucha cups or soaked with kombucha straws; (iv) roasted kombucha cups can fulfill the requirements in drop test, hot-water resistance test, and water leakage resistance test based on GB 180062008. Finally, roasted kombucha straws can pass the bending test and heat endurance test based on ISO18188:2016.

Supervisors: IP Yuen Yu, WONG Tsz Yeung

\section{Marine guard}

TSANG Sung Tak, WONG Tsz Ching, LEE Cheuk Lok, LU Hiu Tung, LAU Cheuk Fai

Christian and Missionary Alliance Sun Kei Secondary School

Marine pollution is a rising problem around the world. It severely affects the ocean including the marine life and their habitats. In the end, the affected marine life could harm humans through the food chain and the polluted habitat may further be harmful. Due to the human involvement, humans should take the matter into their own hands and act to solve it. In order to solve or at least alleviate ocean pollution, we propose to clear the pollutants in the ocean. Therefore, we developed a system, named Marine Guard, that consists of a submarine used for locating pollutants. A camera attached to the submarine records the environment underwater. The system also includes a 3D printed smart buoy for receiving GPS signals and signals from the remote control of the submarine through an antenna. An artificial intelligence (Al) is the core of the system and can recognize pollutants. The system works as follows: users control the submarine to record the environment underwater. The video along with the GPS location from the smart buoy is sent to the Al to detect the pollutants. The Al will specify the location and the type of pollutant on a map. With this information, we can effectively locate the pollutants in the ocean and clean them with ease. Supervisor: CHOW Wing Hei

\section{Invisible hand}

MA Cheuk Yan, LAU Wan Chi, POON Cheuk Ying, CHEUNG Seen Kei Celinda, LEUNG Samantha

Heep Yunn School

The design of the fire escape in our school consists of two fire resistant doors with a small "room" between them. When someone pulls the door, the other door will move automatically. We speculate that the phenomenon is related to air pressure. When we pull the door, the volume between the two doors increases. According to Boyle's Law, an increase in volume causes a decrease in air pressure. Thus, the pressure difference causes the door behind to move automatically. Suppose door A moves by $45^{\circ}$, the volume between the two doors will be increased by $2.4375 \mathrm{~m}^{3}$. As a result, the air 
pressure will drop by $31.3 \mathrm{kPa}$. Shopping malls also have many double-door fire escape designs, but we have not observed this kind of automatic movement of fire doors in shopping malls. After in-depth research, it is found that both sides of the doors in our school are open areas and those in shopping malls are closed areas, as a result, this phenomenon does not occur. Finally, we found that Boyle's law can also be used to improve our daily lives. For example, when the door of a room is opened, the change in air pressure can drive the flow of air, so that the bad smell can be removed in a short time. Our project investigates Boyle's law in 3 different areas: (i) our school, (ii) shopping malls, and (iii) homes, and proposes corresponding applications.

Supervisor: CHOI Tat Fai

\section{Al eye}

\section{TANG King Lung, MOK Siu Wai, CHAN Nok Yin, DU} Yeung

\section{Fanling Rhenish Church Secondary School}

In modern society, many people suffer from chronic diseases due to poor eating habits. Patients need to eat moderately, but they do not know whether the food they choose is suitable for them and eating wrongly may lead to worsening of their condition and even death. We developed a mobile app that allows users to enter the diseases they are suffering from. The scanning function in the app can identify the food in the supermarket to indicate whether the food product is suitable for the user, allow users to choose food that suits them, and help patients recover quickly. We used Google Firebase to create a database with information on diseases and food products such as chocolate that can cause diabetes. Then, we made a 360-degree automatic camera to scan various products from different angles and upload them to the cloud for machine learning. Finally, we developed a food identification app. When a user starts our app to scan an item, the system will use the learned Tensorflow model to detect the item and obtain the item information from the database. The app will show if the product is recommended or not recommended for the user to eat according to the user's disease information. As long as our invention scans the food with the mobile phone, it will immediately show whether the food is suitable for the user. It is more convenient and quicker than searching on the Internet, saving people's time to choose food.

Supervisor: SOO Chi Kin

\section{Waste collection point}

\section{YUEN Hei Pui, HUANG Tsun Hei, KWAN Tsz Hin, LI Sin Tung}

Man Kwan Pak Kau College

Household, food, and paper waste is often not properly sorted in recycling bins. Therefore, it fails to achieve effective classification and is an obstacle to recycling or the reuse of waste. Finally, the garbage sent to landfills or incinerators contributes to pollution. The garbage bin "Waste Collection Point" designed by our team is smaller than the three-color recycling garbage bin. It has a smart identification lens, which applies artificial intelligence (Al) technology, and four classification compartments inside. When someone throws garbage into the Waste Collection Point, the lens automatically identifies the type of garbage and sort it into the corresponding bin. The team's design was first tested and optimized on campus and then promoted to urban use later. The Waste Collection Point uses a lens to identify objects, Micro:bit and MuseLab for programming to establish an "Al intelligent identification" model. First, we prepared the photos of "Bottles", "Drinks", "Cans", and "Others" which are common wastes on campus. These photos were used for model training, and finally for data testing. The training data set provided parameters for the learning model by artificial intelligence, and the effect of the model was evaluated through testing. A servo motor was installed in the "Waste Collection Point". When the intelligent identification model is output, the servo motor receives an instruction to send the garbage into the corresponding garbage bin. Several tests were carried out to adjust the degree of the classification board for effective classification. We hope that the Waste Collection Point will be further optimized and become more humane. The Waste Collection Point may replace regular garbage bins and help to raise the public's awareness of "recycling" habit. We are planning to build in a reward function that allows citizens who use mobile phones to exchange points and promote their willingness to recycle properly.

Supervisor: CHEUNG Kit Yi 


\section{The new biodegradable filter for face mask}

LEUNG Lok Hang Max, LAU Kam Yiu, FUNG King Ho, Oscar, CHAN Orlando, YU Ting Hin, CHEUNG Ka Yu, CHEN Ka Po, HUANG Hoi Fei, YIP Wai Yu

Lung Kong World Federation School Limited Lau Wong Fat Secondary School

Wearing surgical masks is effective in reducing the rate of COVID-19 transmission via respiratory droplets [1]. However, the most popular face mask - surgical mask - is made of non-biodegradable plastics. It technically can only be used once. According to a report in the Environmental Science and Technology journal, there are 129 billion face masks being disposed [2]. This causes serious land and water pollution. Hence, our project aims to make an efficient and environmentally friendly face mask filter - SCOBY filter - to fight against diseases caused by transmission via respiratory droplets. In our study, we successfully developed a SCOBY filter, a membrane peeled from the Kombucha-grown SCOBY. Its physical properties were compared with three other mask filters (surgical mask, reusable mask, and cloth mask filters). We found the SCOBY filter had much better filtration efficiency than the other three mask filters, and its water vapour permeability is comparable to the surgical mask. We also used the count plate method to study the bacterial content of different mask filters. From the results, the SCOBY filter can be sterilized by simply immersing it in boiled water for 5 minutes. This filter is reusable for 28 times once sterilized. We also found the SCOBY filter with one-time sterilization has a shelf life of $\mathbf{3 . 5}$ months. Hence, the SCOBY filter is an efficient and environmentally friendly face mask. Supervisors: CHAN Pik Ying, LI Wing Yan, KEUNG Kwok Hung, LAU Ying Kit

\section{A portable Hong Kong sign language translation platform with deep learning}

Regin LO, CHAN Sze Mga, CHAN Tsz Ying, CHEUNG Hoi Yan, WOO Wing Chai

Christian and Missionary Alliance Sun Kei Secondary School

In Hong Kong, more than 155.000 people are deaf or hard of hearing and use Hong Kong Sign Language for daily communication. Thus, a portable Hong Kong sign language translation platform was developed. With this platform, non-disabled people can easily and quickly translate and understand the sign performed by deaf people through mobile phones. We believe that this platform can facilitate daily communication between deaf people and others in Hong Kong. To use this platform, the mobile phone should be connected to our Jetson Nano. Users will then start recording videos of sign language and the recorded videos will be preprocessed. Preprocessed frames are transmitted to Jetson Nano after the recorded video is sent. In the end, the translation results will be displayed on the screen. To prove the effectiveness of the proposed Hong Kong Sign Language Translation Platform, an experiment was conducted to test the performance in terms of both accuracy and time. In this experiment, 30 sign videos were randomly selected from the testing set of our dataset as the input sign videos. And the average total translation time and accuracy are 5.82s and $93.3 \%$, respectively. In the future, we aim to enlarge the vocabulary size of sign languages.

Supervisor: CHOW Wing Hei

\section{Turning waste into bio-source plastics}

YIM Yui Long, LIANG Kam Lam, YIP Ho Yin, Kwok Tak Seng Catholic Secondary School

The pressure of Hong Kong's landfill has been skyrocketing in recent years due to the production of daily food waste. In addition, the rise in consumerism and the pandemic lead to increased use of plastic products. Hong Kongers discarded 6.000 tonnes of food waste and plastics in 2019, which accounts for half of the municipal solid waste. With an eye to address the issue, we produced bioplastics using the four most commonly found food wastes (boiled rice, orange peel, tea, and sugarcane), together with adding vinegar, glycerine, $\mathrm{Ca} 2+(\mathrm{aq})$, gelatine, and eggshells by following the scientific method proposed by MIT. We produced various bioplastics (with the proportion of food waste to boiled rice 1:1 to 1:7) and tested them with different physical tests, including biodegradability, tensile strength, waterproof test, the time to get mouldy, and toughness. The results show that the bioplastics including eggshell are suitable for replacing some tough plastics while those including $\mathrm{Ca} 2+(\mathrm{aq})$ favours the production of soft plastics. Concerning the experimental results, we produced some bioplastics such as the fruit 
protection net and plastic box. In the future, bioplastics may also be used to replace the traditional plastics which are made from polymers.

Supervisor: WONG Yuk Yee

\section{Genetically modified biosensor for quantitative diagnostic detection of dioxins}

\section{CHENG Sze Lut, CHIU San Bo \\ Pui Ching Middle School}

Dioxin, 2,3,7,8-tetrachlorodibenzo-p-dioxin (TCDD), is toxic or even fatal to both humans and aquatic lives. There are increasing concerns of the bioaccumulation of dioxin in the food chains and ecosystems. However, there are only a few ways to detect dioxin efficiently. This study aims to develop a new way for quantitative diagnostic detection of dioxin using a genetically modified biosensor with high efficiency for practical use. While much research has focused on the use and treats of the aryl hydrocarbon receptor (AhR) pathway in animals, we focus on the potential usage of the AhR pathway in prokaryotes. The DNA-binding action of the AhR complex, which activates the expression of genes following the xenobiotic response element (XRE), can be a diagnostic tool to detect the presence of dioxin in the surrounding environment. Green fluorescence protein (GFP), known for its high accuracy and convenience in determining expression levels of genetic circuits, was chosen to be the reporter protein. Thus, the amount of dioxin present in the environment was quantified by measuring the intensity of fluorescence emitted by GFP. Modification on E. Coli provides a potential for culturing and further study, allowing the biosensor to adapt in different circumstances.

Supervisor: YEUNG Wai Lok Wesley

\section{Intelligent Chinese medicine box system}

\section{CHAN Kin Wang, ZHANG Ming Tai, TSE Sau Kon, YEUNG Ho Tin \\ Heung To Secondary School (Tseung Kwan O)}

The prescriptions written by Chinese medicine practitioners are often scribbled and difficult to read, which may lead to wrong medical dispensing. Patients have no way to know whether the medicine is correct or not. If this problem exists, patients' health may be jeopardized in the dispensing processes. Therefore, we invented a device called Intelligent Chinese Medicine Box System. The system consists of 2 parts: Prescription Preparation Software and Smart Medicine Box. The Software is written by Microsoft Visual Basic.net and uses RFID technology to let Chinese medicine practitioners input prescription information (medicine name and dosage) in the RFID tag, which takes about 1 minute. Dispensers, who receive the prescription tag, receive the content of the prescription including the name and dosage of the medicine by an RFID reader. The dispensers can then prepare the medicine based on this information. The box is equipped with electronic balances and an LCD-display to provide instructions to dispensers and patients about the dosage of the medicines to ensure that there are no mistakes in dispensing medicine. Our results showed that the work of dispensers became smoother. Possible mistakes are minimized after Intelligent Chinese Medicine Box System is adopted. The dispensing work was also more transparent to patients, such that they were aware of mistakes in the dispensing process. In conclusion, this system can increase the public's confidence in Chinese medicine and make the profession of Chinese medicine further affirmed by society. Supervisor: WONG Tung Shek

\section{Magic Chinese medicine residue}

\section{CHENG Man Hei, KEUNG Shing Yin, LEE Yin Yan Irene, LEUNG Nga Man, WONG Sum Yu \\ Sha Tin Government Secondary School}

In recent years, food shortage occurs in many poor countries around the world. Due to the outbreak of COVID-19, the problem has intensified. To deal with this problem, we set up a series of experiments to investigate the use of Traditional Chinese Medicine Residue (TCMR) as fertilizer. In the experiment, we prepared the boiled TCMR. TCMR was mashed with mortar and pestle, baked, and completely dried out. We mixed the completely dried TCMR with soil and Effective Microorganisms. Then we separated the residue into three plastic boxes with different amounts of Effective Microorganisms $(0 \mathrm{~g}, 5 \mathrm{~g}$, or $10 \mathrm{~g})$. We slightly stirred the soil from day to day to ensure the TCMR can decompose evenly. After TCMR decomposed for several days, we removed parts of the TCMR mixture to conduct $\mathrm{pH}$ value and NPK 
value tests. Also, we used the TCMR mixture with different amounts of Effective Microorganisms, different TCMR\%, and different decomposition duration to plant mung beans. After 20 days, we pulled out those mung beans and measured their heights and weights. After the experiment, we found out that the use of TCMR as a fertilizer is feasible. The incorporation of Effective Microorganisms to speed up the process of decomposition is not necessary if TCMR can be mashed into small pieces beforehand. We suggest letting the TCMR and soil mixture decompose for about 20 days. $5 \%$ to $7 \%$ of TCMR inside the soil would be suitable.

Supervisor: CHAN Man Hin

\section{Fiberastic}

\section{CHENG Survin, CHIN Tsz Yan, BAUTISTA Samant- ha Jane}

\section{Kit Sam Lam Bing Yim Secondary School}

According to recent statistics, humans produce billions of tons of waste every year. The two major sources of waste are food waste and plastic waste. Therefore, we developed Fiberastic, a biodegradable plastic made from fibrous residues. We chose avocado pits, sugarcane bagasse, and coconut pulp to make Fiberastic. As these fibrous residues contain a lot of cellulose, the tensile strength can be greatly enhanced. The biodegradable plastic is also made up of starch, water, and glycerol. We mixed those materials together and heated the mixture to obtain the starch gel. The starch gel was then cooled down to form starch plastics. First, the tensile strength of the plastic was tested by clamping it with weights and kept adding until the plastic broke. The results showed that avocado plastics had the highest tensile strength. We observed that the tensile strength can be increased by adding more avocado pits powder and decreased by adding more glycerol. Another testing was heat degradability. The plastic was boiled for 30 minutes and the remaining plastics were filtered and put in the oven to dry. The dried plastic residues were weighed to compare the decomposition amount. Sugarcane plastic had the highest decomposition amount. To further develop our product Fiberastic, different mixtures of fibers can be used to maximize the tensile strength and heat degradability. Moreover, food waste such as waste rice could replace the starch to make the plastic even more environmentally friendly.
Supervisor: LAU Si Nam 